Mots. Les langages du politique

$87 \mid 2008$

Chrononymes. La politisation du temps

\title{
L'Italie et ses Années de plomb. Usages sociaux et significations politiques d'une dénomination temporelle
}

\section{Carmela Lettieri}

\section{CpenEdition}

\section{Journals}

Édition électronique

URL : https://journals.openedition.org/mots/12032

DOI : $10.4000 /$ mots. 12032

ISSN : 1960-6001

Éditeur

ENS Éditions

\section{Édition imprimée}

Date de publication : 21 juillet 2008

Pagination : 43-55

ISBN : 978-2-271-06685-5

ISSN : 0243-6450

\section{Référence électronique}

Carmela Lettieri, «L'Italie et ses Années de plomb. Usages sociaux et significations politiques d'une dénomination temporelle », Mots. Les langages du politique [En ligne], 87 | 2008, mis en ligne le 21 juillet 2010, consulté le 23 avril 2022. URL : http://journals.openedition.org/mots/12032 ; DOI : https:// doi.org/10.4000/mots.12032 


\section{L'Italie et ses Années de plomb. Usages sociaux et significations politiques d'une dénomination temporelle}

L'expression Anni di piombo (Années de plomb) a été adoptée en Italie pour désigner la période qui a fait suite aux mouvements contestataires de 1968 et de $1969^{1}$. L'origine de ce syntagme n'est cependant pas autochtone puisqu'il est devenu récurrent dans le débat public à partir de la diffusion d'un film allemand, Die Bleierne Zeit, réalisé par Margarethe Von Trotta en 19812. L'usage de l'expression s'est imposé a posteriori pour indiquer, selon les différentes interprétations, une partie ou bien la totalité de la décennie soixantedix. Cette période a été caractérisée par l'émergence de mouvements et de groupes ayant choisi la violence comme forme d'action politique. Elle semble rendre compte adéquatement de formes de violence terroriste très particulières, parce que plus importantes, plus hétérogènes et plus durables que celles qui ont été constatées dans d'autres pays. Un parallèle est naturellement possible avec l'Allemagne de l'Ouest, où l'on utilise la même expression pour désigner la décennie 1970 et qui présente le plus d'analogies avec l'Italie3. Dans le débat allemand, l'usage métaphorique de Blei (plomb) recouvre plusieurs significations, ce terme indiquant tout autant le plomb de la culpabilité que celui de la violence. La génération impliquée dans la vague terroriste avait en effet hérité un sentiment de culpabilité dû à la compromission de la précédente dans le Troisième Reich (Elias, 1991; Vitiello, 2007)4. La

Université de Provence, CAER (Centre aixois d'études romanes), carmela.lettieri@univ-provence.fr

1. Une autre expression consacrée indique la dernière partie de l'année 1969 : l'autunno caldo (l'automne chaud), en référence à des grèves ouvrières. De la même façon, l'appellation biennio rosso (deux années rouges) peut être à la fois utilisée pour 1968-1969 et pour 1919-1920, la phase qui a précédé la naissance du Fascisme. Il s'agit là d'exemples qui montrent la multiplicité, l'ambiguïté et parfois la superposition de ces dénominations temporelles.

2. Ce film a reçu le premier prix du Festival de Venise. Le scénario est inspiré par la vie des sœurs Christiane et Gudrun Ensslin, cette dernière étant l'un des membres de la Bande BaaderMeinhof morts en 1977 dans la prison de Stammheim.

3. Toutefois, alors que ce pays a connu seulement le terrorisme d'extrême gauche de la Fraction Armée Rouge, ce qui fait la particularité du cas italien est la grande diversité d'acteurs et l'hétérogénéité des formes d'action pratiquées.

4. Erinnerunglosigkeit (absence de souvenir) et Trauerarbeit (travail de deuil) sont des termes associés dans les explications psychanalytiques du terrorisme allemand. 
revendication d'une filiation par rapport au combat des résistants qui avaient contribué à la chute du régime fasciste n'est pas non plus totalement absente des discours d'autojustification des terroristes italiens. Certains d'entre eux ont revendiqué une continuité générationnelle à travers le thème de la « Résistance trahie». II n'en reste pas moins qu'en Italie, loin de renvoyer à un psychodrame générationnel, le chrononyme Anni di piombo est censé avant tout exprimer l'intensité de la violences et la durée dans le temps ${ }^{6}$.

Au-delà des interrogations d'ordre sociohistorique que cette période suscite et dans le contexte d'une histoire politique encore en construction, nombreux sont les discours en compétition qui investissent la scène publique : témoignages d'acteurs engagés dans la lutte armée, productions journalistiques, discours savants (historiens, sociologues, politologues) ou demi-savants (journalistes, essayistes, commentateurs politiques attitrés), produits culturels (cinéma, littérature, théâtre). Dans un espace public profondément fragmenté, les différentes arènes dans lesquelles se déploient les échanges symboliques sont définies par la diversité des répertoires et des dispositifs utilisés (Tolomelli, 2006).

Au vu de cette abondante production, une enquête menée sur les publications de la période 1989-2007 révèle les significations politiques implicites dans les différences d'usage linguistique. La façon dont l'expression Anni di piombo s'est imposée petit à petit est ici analysée à partir d'un ensemble d'ouvrages aux statuts différents. Il s'agit de livres d'histoire, d'enquêtes journalistiques, d'essais, de témoignages et d'œuvres de fiction.

Deux hypothèses ont guidé ce travail. La première est que la position des acteurs dans le champ politico-médiatique ou bien, en ce qui concerne les usages savants, dans le champ disciplinaire, contribue à expliquer leurs prises de position. La seconde est que la charge sémantique de l'expression évolue en fonction des enjeux contextuels. Nous allons donc combiner ces deux axes d'analyse, qui concernent les positions des acteurs et la conjoncture, avec les différences de périodisation. Le flou qui persiste en ce qui concerne la date du début et celle de la fin de la période est le signe des enjeux politiques et historiographiques qui produisent l'arbitraire du découpage. C'est pourquoi il convient de préciser les implications que la délimitation chronologique comporte avant de présenter la fonction de ce chrononyme.

5. Il faut souligner qu'en italien, la métaphore du plomb renvoie à l'usage des armes à feu, alors qu'en français, elle évoque plutôt l’idée de poids.

6. En italien, une certaine indécision graphique demeure quant à l'utilisation de la majuscule. Cela est dû en partie aux raisons évoquées dans la présentation de ce dossier, en particulier à la difficulté de distinguer nom propre et nom commun dans le cas de chrononymes construits avec des mots qui sont par eux-mêmes porteurs d'une signification temporelle (années). Il faut signaler aussi une plus grande souplesse de la langue italienne dans ce domaine. 


\section{Les implications historiographiques du découpage}

Bien que le chrononyme Années de plomb renvoie de façon implicite à la décennie soixante-dix, il n'y a pas de véritable consensus quant à la date du début et à celle de la fin de la période. C'est ainsi que des évènements et des dates différents peuvent être indiqués comme déclencheurs : l'attentat de la Piazza Fontana à Milan le 12 décembre 1969 ou bien l'année 1976 avec les premiers meurtres politiques des Brigades Rouges7. Ces choix ne sont pas anodins puisque, selon le terminus a quo choisi, on propose une interprétation différente des causes et des responsabilités judiciaires et politiques. On peut en effet rechercher celles-ci dans les connivences entre une partie de l'appareil d'État et les groupuscules d'extrême droite. On attribue au massacre de 1969 un rôle déterminant dans le choix de la lutte armée par un certain nombre de militants de gauche, évoqué par l'expression «perte de l'innocence» (perdita dell'innocenza). Cela est d'autant plus vrai lorsque l'on songe aux difficultés qui persistent pour reconnaitre les responsabilités de ces évènements qui ouvrent la série d'attentats sanglants (stragi) dans des lieux publics fomentés par les groupes terroristes de droite actifs dans la première moitié des années soixante-dix : une voiture piégée explose le 31 mai 1972 à Peteano et provoque la mort de trois carabiniers; un attentat est perpétré lors d'une manifestation promue par les syndicats à Brescia le 24 mai 1974; une bombe explose sur le train Italicus le 4 août de la même année (Lettieri, 2008).

Tandis que certains font commencer dès 1972, date du meurtre du commissaire Luigi Calabresi ${ }^{8}$, voire 1969 , la radicalisation des mouvements activistes de gauche, pour d'autres elle n'aurait en fait véritablement commencé que vers la moitié de la décennie. Le premier meurtre politique prémédité réalisé par les Brigades rouges est en effet celui du juge Francesco Coco, le 8 juin 1976. On peut reculer ou avancer la date de début du phénomène en fonction de l'interprétation que l'on propose des faits historiques et surtout en fonction de l'analyse que l'on fait des évènements se situant à la charnière des deux décennies soixante et soixante-dix (Fournel, Zancarini, 1997).

7. Le 12 décembre 1969, l'explosion d'une bombe dans le hall de la Banque de l'Agriculture à Milan provoqua 17 morts et 87 blessés. Sur le plan judiciaire, les responsabilités de ce massacre n'ont toujours pas été élucidées. Même si, au lendemain de l'attentat, l'enquête de la police s'oriente vers les milieux anarchistes et d'extrême gauche, ces derniers dénoncent un «massacre d’État» (strage di stato). Il a été établi en effet que cet évènement a marqué la consolidation de la «stratégie de la tension » dont le but était de provoquer un tournant autoritaire.

8. Trois ex-militants de Lotta continua, Adriano Sofri, Ovidio Bompressi et Giorgio Pietrostefani ont été condamnés pour cet assassinat. L’issue de leur parcours judiciaire a cependant provoqué de nombreuses réactions parmi les intellectuels et tous ceux qui, en Italie et ailleurs, sont convaincus de leur innocence (Ginzburg, 1997). 
Il en est de même pour l'apogée du terrorisme. Pour certains, il s'agit de l'année 1977 (mouvement des indiani metropolitani), pour d'autres de l'enlèvement et du meurtre d'Aldo Moro, leader de la Démocratie chrétienne, en 1978. Alors que le climat de guérilla urbaine créé par les conflits qui opposent les franges les plus extrêmes s'accentue, cet assassinat marque un tournant dans la stratégie des Brigades rouges. La logique de la clandestinité, les choix stratégiques des nouveaux dirigeants ainsi que la réponse répressive de l'État en accentuent la violence et le caractère militariste (Della Porta, 1985). À tel point que $80 \%$ de l'ensemble des actions des Brigades rouges se situe entre 1978 et 1980.

Les mêmes incertitudes persistent quant à la date de fin : le 7 avril 1979, l'attentat à la gare de Bologne en août 1980 et l'année 1982 sont tour à tour indiqués comme terminus ad quem. En effet, suite à un gigantesque coup de filet organisé à l'université de Padoue qui, selon les enquêteurs, abrite des agitateurs proches des groupes armés - parmi lesquels le philosophe Toni Negri - de nombreux militants vont quitter le pays pour se réfugier à l'étranger, notamment en France. Par ailleurs, la bombe qui explose dans le hall de la gare de Bologne le 2 aout 1980 n'est pas le dernier massacre perpétré par les groupes d'extrême droite, mais il est sans doute le plus meurtrier : 85 morts et 200 blessés9. Les derniers leaders des Brigades rouges (Mario Moretti, Giovanni Senzani) sont arrêtés en 1981-1982. De nombreux procès s'ouvrent après les lois sur la dissociation et sur les repentis, ce qui permet le démantèlement des réseaux (Sommier, 2000); mais les groupes d'extrême gauche (Brigate Rosse, Prima Linea, Nuclei armati proletari) continuent de sévir pendant la décennie suivante ${ }^{10}$. Si on ajoute à cela les nombreuses suites judiciaires, les procès qui se prolongent sur plusieurs décennies et dont les issues sont sujettes à caution, on comprend la difficulté de déterminer objectivement une fin pour cette période si conflictuelle de l’histoire italienne.

\section{L’évolution des emplois du chrononyme}

\section{Une conjoncture fluide (1969-1984)}

Dans les années où ces évènements se produisent, des locutions très politisées et qui marquent donc un jugement manifeste sont en concurrence pour

9. Giusva Fioravanti et Francesca Mambro ont été condamnés en 1995 pour en avoir été les exécuteurs matériels. Quelques années plus tard, le 23 décembre 1984, une énième bombe explose dans le tunnel qui relie Florence à Bologne, que traversait le train Italicus, en provoquant 16 morts et 130 blessés.

10. Il suffit de citer l'enlèvement du général de l'Armée des États-Unis James Lee Dozier, le 17 décembre 1981, l'assassinat de l'économiste Ezio Tarantelli, le 27 mars 1985 et le meurtre de l'ex-maire de Florence, Lando Conti, le 10 février 1986. 
désigner les éléments du contexte politique. En impliquant des prises de position évidentes, le langage politique utilisé entend du même coup infléchir tout autant la lecture que le cours des évènements. C'est par exemple le cas de l'utilisation, dès la fin de l'année 1969, de l'expression strategia della tensione ${ }^{11}$.

Même le mot terrorisme n'est utilisé dans le débat public et dans les médias qu'à partir de la moitié des années soixante-dix. À l'époque, soit la violence politique est volontairement occultée dans le langage (Lettieri, 2000), soit on y fait référence par des termes connotés : estremismo (extrémisme), eversione (subversion), squadrismo (emploi des équipes d'action) (Della Porta, 1990). On fait alors appel à des cadres d'interprétation connus, puisque, par exemple, ce dernier désignant rappelle les formes de violence en vogue pendant le fascisme. De plus, la Démocratie chrétienne saisit l'occasion pour imposer une interprétation dont ce parti peut tirer bénéfice, en affirmant son rôle de médiation face aux dérives des «extrémismes opposés » (opposti estremismi). Cette locution suggère que les idéologies de droite et de gauche impliquent le risque de dérives violentes et antidémocratiques, ce qui fournit une justification à la politique centriste du gouvernement.

Pendant la première partie de la décennie quatre-vingt, lorsque se produit le passage à une économie postfordiste, l'affirmation d'un libéralisme accentué passe par l'abandon de toute référence linguistique aux différentes versions de l'ouvriérisme et aux conflits idéologiques ancrés dans le monde du travail, notamment dans celui des usines (Lumley, 1998). Les communiqués des Brigades rouges, qui ont été largement diffusés et ont alimenté la discussion, n'ont pas peu contribué à dévaloriser un type de langage politique - dont le terme sinistrese (jargon de la gauche) rend compte - assimilé à un véritable délire idéologique.

\section{La phase d'incubation du chrononyme (1984-1991)}

La «normalisation» des années suivantes, lorsque les actions terroristes se raréfient, crée également un terreau propice au changement des habitudes linguistiques. C'est alors que le chrononyme Anni di piombo entre progressivement dans l'usage. Il convient toutefois de distinguer l'emploi qui en est fait en fonction du type de texte et de locuteur. Une première distinction peut être établie entre les usages savants, ceux des historiens, notamment,

11. Le phénomène que l'on indique par cette expression désormais consacrée, dont l'origine remonte à un article de l'hebdomadaire britannique The Observer du 14 décembre 1969, a été analysé par Franco Ferraresi. Cet historien en indique les principaux acteurs : la droite radicale, les services secrets, la police, la magistrature. Il met également en garde sur le sens à donner au terme strategia. Plus que d'un véritable complot, il s'agissait des effets d'un climat politique : les acteurs impliqués n'agissaient pas nécessairement en suivant un plan défini ni en accord entre eux (Ferraresi, 1995). 
et l'ensemble hétérogène des discours des essayistes et/ou des journalistes qui écrivent des ouvrages de vulgarisation, des enquêtes ou des témoignages. Un nombre important de ces publications inclut cette formule dans le péritexte, notamment dans les titres, installant la lecture à l'intérieur d'un horizon de réception déterminé (Chartier, 1997 ; Mouillaud, 1993) ${ }^{12}$.

Le livre publié par les journalistes Indro Montanelli et Mario Cervi en 1991 est l'archétype de la production qu'on peut dire «semi-savante». Il s'agit de la première publication comportant le syntagme Anni di piombo dans le titre et l'utilisant comme étiquette pour désigner une période de l'histoire de l'Italie. Dans la périodisation proposée, due sans doute aux exigences éditoriales, cette phase est close en 1978 par l'élection de Sandro Pertini (PS) à la présidence de la République. C'est sans doute ce genre de publications, dont les auteurs revendiquent leur rôle de "témoins non neutres», qui est à l'origine de l'introduction du chrononyme dans l'usage courant, car elles ont rencontré un large succès ${ }^{13}$. À mi-chemin entre cette catégorie et les ouvrages scientifiques, se situent les publications à visée vulgarisatrice signées par Giorgio Galli14. Ici, la rigueur de l'enquête laisse parfois la place à une forte implication de l'auteur, ce qui rend le classement ardu.

Alors que dans le cadre de disciplines comme la sociologie ou la science politique, la locution Anni di piombo n'est pas employée, chez les historiens on constate au départ des réticences qui seront abandonnées. La nouvelle génération de chercheurs adopte le chrononyme avec beaucoup moins de réserves; il faut dire qu'elle a une formation moins classique et qu'elle est sensible à l'histoire sociale et culturelle (Simona Colarizi, Stefano Pivato, Paul Ginsborg). De plus, ces historiens ont réalisé leur socialisation politique pendant cette période.

En France, l'expression est utilisée dans des titres de thèses ou de mémoires de recherche à partir des années quatre-vingt. Son choix est révé-

12. Le premier ouvrage qui comporte l'expression dans le titre d'un chapitre parait en 1989 (voir en annexe).

13. Indro Montanelli (1909-2001) a été l'un des plus célèbres journalistes italiens. Éditorialiste du quotidien Corriere della Sera depuis les années trente jusqu'en 1973, puis entre 1995 et 2001, il a également lancé deux quotidiens : /l Giornale (1974-1994) et La Voce (1994-1995). Ex-fasciste déçu par le régime de Mussolini, conservateur et anti-communiste, il fut blessé aux jambes par un commando des Brigades rouges en juin 1977. Cette expérience a sans doute marqué sa perception de la période qualifiée, dans la préface de son ouvrage, de «tragique» - «les années les plus sombres de l'histoire italienne». Cet auteur met également en avant la méthode utilisée : «Ayant vécu ces années à la première personne en tant que journaliste et commentateur, nous n'avons pas eu besoin de consulter beaucoup de textes ou de documents, nos souvenirs étaient suffisants. » La série en plusieurs volumes consacrée à l'histoire italienne qu'il a publiée chez Rizzoli est l'un des best-sellers de l'édition italienne (plus d'un million d'exemplaires vendus). C'est ainsi qu'il est passé du rôle de témoin à celui de «conscience morale» du pays.

14. Giorgio Galli, né en 1928, est politologue et essayiste. Spécialiste des partis poliques, il a publié ses enquêtes sur la corruption, la mafia et le pouvoir politique chez Kaos (Milan), éditeur spécialisé dans les ouvrages de critique sociale. 
lateur du regard porté sur une réalité externe et étrangère mal connue, d'où le besoin d'une clé d'interprétation univoque ${ }^{15}$. Mais si l'emploi du chrononyme fonctionne comme un mode totalisant d'appréhension d'un contexte, il contient un risque de simplification en réduisant la réalité italienne de ces années-là à la seule violence terroriste.

\section{La banalisation du chrononyme (1997-2006)}

Après une période d'accalmie, une véritable effervescence éditoriale se produit à partir de la fin des années quatre-vingt-dix (voir titres en annexe). Outre un intérêt renouvelé de la part des historiens, de nombreux témoignages et ouvrages de fiction voient le jour. Parfois, ce sont les ex-terroristes eux-mêmes qui relatent leur expérience par le biais de romans ou de récits fictionnels, ce qui produit l'exploitation d'un potentiel esthétique d'aventures dont les terroristes et les victimes deviennent parfois les héros romantiques (Dai Prà, 2005; Paolin, 2006; Lettieri, 2006).

En ce qui concerne les témoignages des anciens brigadistes, il est rare que les protagonistes qui ont joué un rôle important dans l'organisation clandestine recourent à l'expression Anni di piombo ${ }^{16}$. En revanche, un besoin de reconnaissance peut expliquer son utilisation de la part de personnages secondaires voulant échapper à un rôle de simples figurants de la lutte armée. Par ailleurs, des témoins qui ont vécu les évènements conflictuels dans le camp opposé publient également leurversion des faits : le préfet Achille Serra, l'ambassadeur des États-Unis Richard Gardner, l'agent des services secrets Fabrizio Grignetti (voir liste des titres en annexe).

Les publications dont le titre contient l'expression Anni di piombo deviennent alors pléthoriques. Un indice de cette véritable mode éditoriale est le sort de la traduction italienne d'un ouvrage de Robert Lumley paru en anglais en 1994 : States of Emergency. Culture of Revolt in Italy from 1968 to

15. Il s'agit surtout de travaux réalisés en science politique et en histoire. À titre d'exemples, dès 1985, Raoul Girardet a dirigé un mémoire de science politique : Glicenstein (Gilles), Viser le cœur de l'État. L'Italie terroriste des années de plomb, IEP de Paris. Plus récemment, Ugo Emprin a soutenu un mémoire à l'IEP de Grenoble : Les fantômes de Bologne. Histoire et mémoire des années de plomb dans la ville rouge, sous la direction de l'historien Jean-Paul Burdy.

16. Les leaders des Brigades rouges ont tous publié des autobiographies ou des témoignages qui ont parfois connu une deuxième édition : Renato Curcio (avec Mario Scialoja), 1995, A viso aperto, Milan, Mondadori ; Valerio Morucci, 2004, La peggio gioventù, Milan, Rizzoli ; Mario Moretti (avec Carla Mosca et Rossana Rossanda), 1994, Brigate Rosse. Una storia italiana, Milan, Anabasi, rééd. 2002, Baldini-Castoldi-Dalai; Anna Laura Braghetti (avec Paolo Tavella), 1998, Il prigioniero, Milan, Mondadori, rééd. 2003, Feltrinelli ; Barbara Balzerani, 1998, Compagna Luna, Milan, Feltrinelli; Alberto Franceschini (avec Giovanni Fasanella), 2004, Che cosa sono le $B R$, Milan, BUR. Ce dernier est aussi le seul à avoir publié en 1997 un livre à mi-chemin entre le témoignage et la fiction, qui comporte le chrononyme dans le titre (voir en annexe). 
1978. Pour les nécessités du marché, le titre italien reproduit l'étiquette que l'on a désormais collée à cette période : Dal'68 agli anni di piombo. Studenti e operai nella crisi italiana.

De nombreux romans et témoignages ne comportent pas l'expression dans sa totalité, mais uniquement le terme allusif piombo. Souvent le syntagme Anni di piombo est contenu dans le titre. Lorsqu'il fait partie du soustitre, il parait jouer le rôle d'une explicitation mais le renvoi à la période, sans autre explication, conduit à le vider de sens. Certains de ces livres sont en effet consacrés à des sujets qui n'ont qu'un lien indirect avec le contexte politique : le sport (In bianco e nero. Una grande Juve negli anni di piombo); la religion (Teresilla. La suora degli anni di piombo); la critique littéraire (Un po' prima del piombo). On peut alors formuler l'hypothèse d'une d'inflation langagière consécutive à la perte de la charge sémantique de la locution.

\section{L'usage du chrononyme dans la presse quotidienne}

L'analyse des articles publiés par le quotidien La Repubblica entre 1984 et 2006 confirme la même évolution des usages. Deux périodes ressortent en raison d'une fréquence accrue du chrononyme dans les titres : 1984-1991 (phase d'incubation) et 1997-2006 (phase de banalisation).

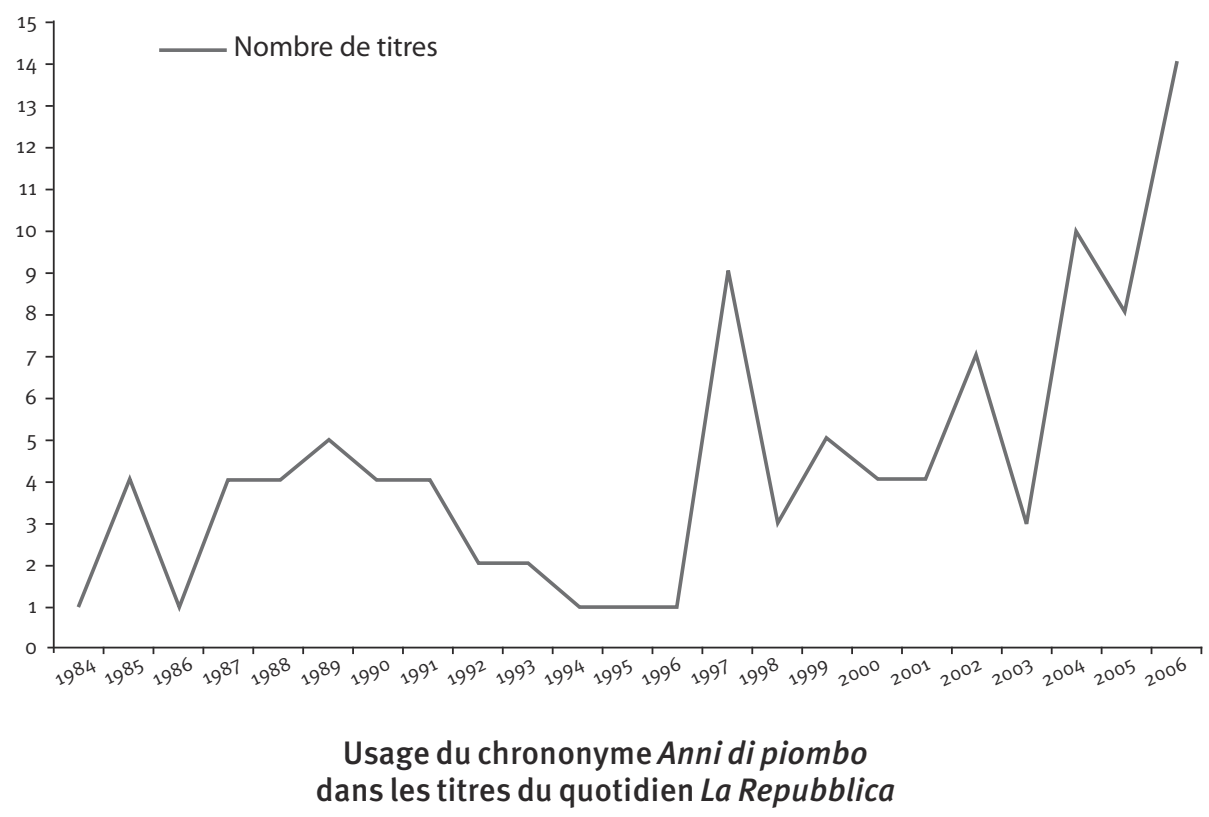


À noter que les articles publiés tout au long de la décennie quatre-vingt concernent surtout les suites judiciaires des actes de terrorisme. En effet, pendant ces années, ont lieu les procès des groupes clandestins, de droite comme de gauche, en particulier les différents procès Moro. La proposition d'une amnistie vient compliquer le débat autour de cette période. Un certain nombre d'articles repérés relèvent de la critique cinématographique ou des commentaires sur les émissions de télévision. Il en est ainsi à l'occasion de la sortie du film réalisé par Bernardo Bertolucci (Segreti, segreti) en 1985, ou bien lorsque la Rai diffuse un reportage du journaliste Sergio Zavoli (La notte della Repubblica) en 1989.

Les thématiques liées à la période précédente passent au second plan entre 1992 et 1996 face à l'actualité politique : les enquêtes des juges de Mani pulite en 1992, la crise de l'ancien système de partis, l'arrivée au pouvoir de Silvio Berlusconi en 1994. Le pic de l'année 1997 s'explique par la discussion autour d'un projet de loi d'amnistie présenté par le ministre de la Justice Giovanni Maria Flick et par le retour en Italie de Toni Negri. La reprise et la banalisation au cours des dernières années sont confirmées par la fréquence de l'expression dans un nombre d'articles de plus en plus élevé. Il s'agit de chroniques judiciaires, d'articles relatifs à l'amnistie et aux repentis, mais aussi à divers sujets sans lien avec la politique : comptes rendus de films (lors de la sortie du film Buongiorno Notte, réalisé par Marco Bellocchio, par exemple), de livres, de concerts, d'expositions ou de manifestations culturelles. Les Années de plomb sont en effet devenues matière privilégiée pour des produits culturels de plus en plus variés.

Alors que les médias sont souvent tenus pour responsables des dérives langagières, les données empiriques suggèrent en revanche une certaine réactivité par rapport aux productions culturelles dont la presse rend compte et vis-à-vis du contexte politique dans lequel elle intervient.

Le souvenir de cette période si conflictuelle de l'histoire récente de l'Italie ne va pas sans problème. Il s'agit en effet d'une mémoire encore sensible puisque le débat public se déroule essentiellement sur le plan judiciaire et reproduit souvent tels quels les anciennes fractures et les clivages politiques de l'époque.

Les mécanismes complexes de construction de la mémoire collective passent par la compétition entre les discours qui évoquent, décrivent ou analysent les évènements historiques. À travers ce procédé de représentation linguistique, qui contribue à la construction symbolique de la réalité, certains discours deviennent, à un moment donné, des discours dominants et réussissent à imposer leur propre vision.

Mais la multiplication des emplois, qui induit la perte de connotation des termes et des expressions, est en même temps le produit d'un processus de 
normalisation et la cause d'un réductionnisme évident. Il va de soi qu'il s'agit, plutôt que de responsabilités individuelles, d'un concours de circonstances et d'une convergence d'intérêts entre des commentateurs politiques, des témoins, des acteurs et des essayistes, qui facilitent une lecture univoque, globale et sans nuances de la période. L'habitude d'évoquer cette période de crise par une formule uniforme et totalisante n'est pas dénuée de risques: l'usage accru de ce chrononyme, dans son apparente efficacité, risque de produire une vision simpliste et de rabattre ces années sur une seule dimension, celle de la violence (Milanesi, 2006). Certes, un chrononyme est par nature réductionniste : le problème essentiel est celui du choix de l'élément auquel on réduit la période ainsi désignée. Si l'issue de ces luttes de labellisation est l'accent mis sur la diffusion de la violence, il en découle l'occultation des causes de cette même violence et des effets d'autres phénomènes tout aussi importants. Car au-delà des répertoires d'action utilisés, les mouvements sociaux des années soixante-dix ont permis l'entrée de nouveaux acteurs dans le champ politique - les femmes et les jeunes par exemple - en produisant des effets non négligeables sur la structure de l'offre partisane et sur les formes de la participation politique dans les années suivantes.

\section{Références}

ChARTIER Roger, 1997, «Du livre au lire», P. Beaud, P. Flichy, D. Pasquier, L. Quéré éd., Sociologie de la communication, Paris, Réseaux-CNET, p. 271-290.

CRAINz Guido, 2003, Un paese mancato. Dal miracolo economico agli anni ottanta, Rome, Donzelli.

DAI PRÀ Silvia, 2005, "Lo sterminato romanzo degli anni settanta », Lo straniero, $n^{\circ}$ 60, juin.

DelLa Porta Donatella, Rossı Maurizio, 1985, «I terrorismi in Italia tra il 1969 e il 1982 », G. Pasquino éd., Il sistema politico italiano, Bologne, II Mulino, p. 418-456.

Della PorTA Donatella, 1990, "Gli anni del terrorismo », Storia della società italiana, Milan, Teti editore, vol. 25, p. 11-59.

- 1990, Il terrorismo di sinistra, Bologne, Il Mulino.

- 1995, «ll terrorismo», Dizionario di politica italiana, G. Pasquino éd., Rome, Bari, Laterza, p. 535-548.

- 1995, Social Movements, Political Violence and The State, Cambridge, Cambridge University Press.

ELIAS Norbert, 1991, Itedeschi. Lotte dipotere ed evoluzione dei costumineisecolixIxexx, Bologne, Il Mulino.

FERRARESI Franco, 1995, Minacce alla democrazia. La destra radicale e la strategia della tensione, Milan, Feltrinelli. 
Fournel Jean-Louis, ZANCARINI Jean-Claude, 1997, "Des historiens peu prudents. L'enjeu historiographique de l'affaire Bompressi, Pietrostefani, Sofri», Les Temps modernes, $n^{\circ}$ 596, novembre-décembre, p. 174-192.

GinzBURG Carlo, 1991, Il giudice e lo storico. Considerazioni in margine al processo Sofri, Turin, Einaudi (traduction française : 1997, Le juge et l'historien, Paris, Verdier).

LeTtIERI Carmela, 2008, L'Italie contemporaine, Paris, Ellipses.

- 2006, «ll corpo dell'inglese de G. Simi. Le noir comme révélateur des sédiments de l'histoire », Cahiers d'études romanes, $n^{\circ} 15$ / 1, p. 171-183.

- 2000, "Anni di piombo et Seconda Repubblica. Les usages linguistiques dans le quotidien Corriere della Sera : une analyse diachronique», Italie. Années quatrevingt-dix, $\mathrm{n}^{\circ} 20$, p. 231-252.

LUMLEY Robert, 1998, Dal ‘68 agli anni di piombo. Studenti e operai nella crisi italiana, Milan, Giunti (1're édition : 1994, States of Emergency. Culture of Revolt in Italy from 1968 to 1978 , Londres, Verso).

Mouillaud Maurice, 1982, "Grammaire et idéologie du titre du journal», Mots. Les langages du politique, $\mathrm{n}^{\circ} 4$, mars, p. 69-91.

MILANESI Claudio, 2006, "Le mouvement vu de l'intérieur: Milan de 68 aux années de plomb à travers le roman d'un témoin", Actes du colloque La valeur de la littérature après les années 1970, Igitur, Université d'Utrecht, p. 408-422.

PAOLIN Demetrio, 2006, Una tragedia negata. Il racconto degli anni di piombo nella narrativa italiana, vibrisselibri.net

Pellegrino Giovanni, Fasanella Giovanni, Sestrieri Claudio, 2000, Segreto di Stato, Turin, Einaudi.

SOMmIER Isabelle, 1998, La violence politique et son deuil. L'après 68 en France et en Italie, Rennes, Presses universitaires de Rennes.

- 2000, «Repentir et dissociation : la fin des années de plomb en Italie?», Cultures \& Conflits, $\mathrm{n}^{\circ} 40 / 4, \mathrm{p} .43-61$.

TARrow Sidney, 1990, Democrazia e disordine. Movimenti di protesta e politica in Italia (1965-1975), Rome, Bari, Laterza.

Tolomelu Marica, 2006, Terrorismo e società. Il pubblico dibattito in Italia e in Germania negli anni settanta, Bologne, II Mulino.

TRANFAglia Nicola, 1997, «Un capitolo del doppio stato. La stagione delle stragi e dei terrorismi (1969-1984)", Storia dell'Italia Repubblicana, vol. 3, Turin, Einaudi, p. 7-80.

Vitiello Guido, 2007, «L'autunno tedesco e l'ombra lunga di Hitler», C. Uva éd., Schermi di piombo. Il terrorismo nel cinema italiano, Soveria Mannelli, Rubbettino, p. $185-207$. 


\section{Annexe}

\section{Utilisation de l'expression dans les titres des ouvrages partype de discours (1989-2007)}

\section{Ouvrages savants (8)}

Ginsborg Paul, 1989, Storia d'Italia dal dopoguerra ad oggi, Turin, Einaudi [titre de chapitre].

Monteleone Franco, 1992, Storia della radio e della televisione in Italia, Venise, Marsilio [titre de chapitre].

LUMLEY Robert, 1998, Dal'68 agli anni di piombo. Studenti e operai nella crisi italiana, Florence, Giunti.

ColArIzI Simona, 2000, Storia del Novecento italiano, Milan, BUR [titre de chapitre].

PIVATo Stefano, 2005, Bella ciao. Canto e politica nella storia d'Italia, Rome, Bari, Laterza [titre de chapitre].

Magnaninı Claudia, 2006, Autunno caldo e anni di piombo. Il sindacato milanese dinanzi alla crisi economica e istituzionale, Milan, Angeli.

CRISTAdoro Nicola, 2006, Eversione di destra negli anni di piombo, Collegno, Chiaramonte.

Uva Christian, 2007, Schermi di piombo. Il terrorismo nel cinema italiano, Soveria Mannelli, Rubbettino.

\section{Vulgarisation (7)}

Montanelli Indro et Cervi Mario, 1991, L'Italia degli anni di piombo, Milan, Rizzoli.

GALLI Giorgio, 1993, Il partito armato. Gli anni di piombo in Italia (1968-1986), Milan, Kaos.

Adriano Pino et CIngolanı Giorgio, 2000, Corpi di reato. Quattro storie degli anni di piombo, Milan, Costa \& Nolan.

FRANCHI Paolo et INTINI Ugo, 2005, Le parole di piombo. Walter Tobagi, la sinistra e gli anni del terrorismo, Rome, Mondoperaio.

Galli Giorgio, 2005, Piombo rosso. La storia completa della lotta armata in Italia dal 1970 ad oggi, Milan, Baldini Castoldi Dalai.

Telese Luca, 2006, Cuori neri. Dal rogo di Primavalle alla morte di Ramelli. 21 delitti dimenticati degli anni di piombo, Milan, Sperling \& Kupfer.

GUIDELLI Giorgio, 2006, Terra di piombo, Urbino, Quattroventi.

\section{Témoignages (11)}

MARINo Leonardo, 1992, La verità di piombo, Milan, Ares.

GARBOLI Cesare, 1998, Un po' prima del piombo, Milan, Sansoni. 
Grignetti Francesco, 2002, Professione spia. Dal fascismo agli anni di piombo, cinquant'anni al servizio del $K G B$, Venise, Marsilio.

PARODI Mario, 2003, In bianco e nero. Una grande Juve negli anni di piombo, Turin, Bradipolibri.

GARDNERRichardR., 2004, Mission:Italy. Gliannidipiomboraccontatidall'ambasciatore americano a Roma (1977-1981), Milan, Mondadori.

Cavallina Arrigo, 2005, La piccola tenda azzurra che i prigionieri chiamano cielo. Anni di piombo, carcere e ricerca di identità, Milan, Ares.

CASAmassima Pino, 2005, Donne di piombo : undici vite nella lotta armata, Milan, Bevivino.

GARIBALDI Luciano, 2005, Com'erano Rosse le mie Brigate : gli anni di piombo visti da un giornalista dalla parte sbagliata, Rome, Nuove idee.

Serra Achille, 2006, Poliziotto senza pistola. A Milano negli anni di piombo e della malavita organizzata, Milan, Bompiani.

Valle Annachiara, 2006, Teresilla. La suora degli anni di piombo, Milan, Paoline.

VECCHIO Concetto, 2007, Ali di piombo, Milan, Rizzoli.

\section{Fiction (7)}

Crosato Francesco, 1993, Anni di piombo in ospedale, Trente, New Magazine.

FrANCESCHINI Alberto et SAmueli Anna, 1997, La borsa del presidente. Ritorno agli anni di piombo, Rome, Ediesse.

D’Amore Antonio, 2001, Una vita sotto torchio. Le straordinarie avventure di Luigi Maestri e dei suoi ottanta... anni di piombo, Milan, Mediamed.

Barbaranelli Fabrizio, 2001, Prigionieri di un incubo. Glianni di piombo della giustizia, Rome, Gangemi.

DelPIANo Grazia, 2004, I miei anni di piombo, Bergame, Antares.

Cimatti Duccio, 2005, Piombo, Milan, Piemme.

PARISI Paolo, 2006, Il sequestro Moro. Storie dagli anni di piombo, Padoue, Becco Giallo. 